\title{
Feed-forward control for a Drinking Water Treatment Plant chlorination process
}

\author{
${ }^{1,3}$ J. Gamiz, ${ }^{2}$ H. Mart'nez ${ }^{1}$ A. Grau, ${ }^{1}$ Y. Bolea \\ ${ }^{1}$ Automatic Control Dept. \\ Universitat Politecnica de Catalunya, UPC, Barcelona \\ ${ }^{2}$ Department of Electronic Engineering \\ Universitat Politecnica de Catalunya, UPC, Barcelona \\ ${ }^{3}$ Dept. de Sistemas de Control Industrial de Aguas de Barcelona \\ Aigües de Barcelona, Barcelona \\ javier.gamiz; herminio.martinez;antoni.grau;yolanda.bolea@upc.edu
}

\author{
3 R. Vilanova \\ ${ }^{3}$ Department of Telecommunications \\ and Systems Engineering \\ Universitat Autonoma de Barcelona \\ 08193 Bellaterra, Barcelona
}

Ramon.Vilanova@uab.cat

\begin{abstract}
Chlorination in drinking water treatment plants (DWTP) is the nal process applied to water before it is sent to storage tanks in the supply network for subsequent human consumption. An excessive dosage of chlorine or, conversely, too small a dosage, may breach existing legal regulations on mandatory limits. In DWTP where there is no signi cant variability in the quality of the water to be treated, a type of control that is proportional to the ow rate in the ef uent can have fully satisfactory results. Therefore, Proportional-Integral (PI) control is a rather frequently used solution. However, when there are inherently long delays in the process, variability in the quality of the water to be treated and considerable variations alternative type is needed. This article presents the strategy and results of a control method that proposes a Fuzzy based feed-forward system to complement an existing PI control. The control system results are shown as applied to the DWTP of Barcelona city, producing satisfactory experimental results.
\end{abstract}

Index Terms-Chlorination Process, Feedforward Control, Fuzzy Control

\section{INTRODUCTION}

Chlorine is the most widely used disinfectant in DWTP due to its high efficiency as a germicide and its excellent capacity to eliminate odors and tastes from water destined for human use. It is also used in Wastewater Treatment Plants (WWTP) to minimize the public health risks associated with exposure to reclaimed water, where the most important goal is the reduction of pathogens during tertiary treatments [1], [2].

Chlorination processes at output from DWTP have been extensively analyzed in terms of control. The interest lies in achieving a fully automated process, in which humans only intervene as supervisors, and in which the quality of the effluent complies with the established regulations, while optimizing operating costs. In the last decade, advances in online instrumentation, [3], [4], [5], i.e. our eyes on the ground, together with controllers with the capacity to run advanced control algorithms, have successfully automated processes that were traditionally controlled manually.

This research was funded by the Spanish MINECO/FEDER grant DPI 2016-77271.
However, in the case of chlorination, it would be no use having the finest instrumentation and controls to address the problem if the chemistry of the water to be treated is not taken into consideration. Most problems in chlorination control come down to an improper understanding of the chemistry of water, inexistent or incomplete modelling, and/or problems derived from a poor choice of sensors or actuators.

The reaction of chlorine when it comes into contact with water can be divided into several phases [6], which clearly mark the control strategy applied. In a first phase, a significant part of the chlorine reacts quickly with the existing (organic and inorganic) matter [7], these reactions occur between the first 5 and 10 minutes [8] after the chlorine comes into contact with the water. The amount of organic matter and nitrogenous substances that react in these first moments will cause considerable variation in the gain of the process in a quick reaction and must be taken into account when designing the control system. When the variability in water quality is homogeneous, this problem will be barely exist. However, when treating waters in which this amount of organic matter can vary, and therefore its chlorine demand too, a control is required with variable gains that adapt to this characteristic. The problem is heightened by the occasional existence of ammonia, which might vary the process gain in a non-linear manner and cause what is called a breakpoint scenario. In these cases, the measurement of residual chlorine at output, the controlled variable, goes through three states with different behaviors. Depending on the nitrogen-derived compound that is reacting in each of these three states, the demand for chlorine will vary. The control system in these cases must be able to deal with these changes in water chemistry and detect when the transition from one state to another occurs [ [9]. This phenomenon is explained in [10] from the point of view of water chemistry, as well as the influence when computing the process gain for the control system.

There are also certain problems that can hinder the control 
of chlorination caused by the sensors and actuators used. Regarding residual chlorine analyzers, it is important to consider their location to ensure that their free chlorine reading is a real value and therefore significant. They must therefore be located somewhere that guarantees that the water to be treated at its highest flow has been in contact with the chlorine for at least $30 \mathrm{~min}$, otherwise the meter reading will not be correct.

This work presents a strategy to control chlorination processes with severe disturbances and long delay times. A feed-forward control system designed on the basis of the operator's experience and data validated by more than 5 years of records is used. The proposed control model can be applied to plants where there is variability in the demand for chlorine, thus providing a method for the design of a type of control system that takes this factor into consideration. It can also be applied to respond to severe disturbances in water chemistry, such as the appearance of ammonia; or operations such as changes between chlorination cabins that are not always linear. Also, as a contribution, based on the variation in flows it offers a potential solution for changes in downtimes that might affect the control system.

One of the advantages of the solution proposed here over other solutions is that it might not require specific hardware for physical implementation. Indeed, this study provides a solution involving an easily implemented control strategy using the existing hardware in the DWPT under study. Specifically, the market controller used is a commercial PLC (Programming Logic Controller) made by Rockwell Automation. Unlike other control systems presented in the literature, this one requires much less computation, is easily exportable to other architectures (since it is based on a standard), and is also robust enough to be used in industrial environments.

\section{Drinking Water Treatment Plant (DWTP)}

Since its creation in 1955 until today, the DWTP of Sant Joan Desp has become one of the most technologically advanced water treatment plants in Europe. With a drinking water production of $120 \mathrm{Hm}^{3} /$ year and a capacity of $6 \mathrm{~m}^{3} / \mathrm{s}$, it provides $58 \%$ of the drinking water that supplies the city of Barcelona. The DWTP that is the object of this article is one of the most technologically advanced water treatment plants in operation. Producing $120 \mathrm{Hm}^{3}$ of drinking water a year and with a maximum capacity of $6 \mathrm{~m}^{3} / \mathrm{s}$, it is one of the biggest drinking water treatment plants in Southern Europe. At this DWTP, traditional processes such as pretreatment, clarification and final disinfection of water coexist alongside other latest generation ones, such as treating water with ozone and active carbon, and reverse osmosis. For disinfection, there are two chlorination tanks, the first of these being the subject of the work presented herein. Figure (1) shows a diagram of tank 1 where the first chlorination of the water to be treated at the DWTP is performed. The $10,000 \mathrm{~m}^{3}$ tank is hydraulically connected to a second tank of identical size and which, in the event of an anomaly in either of these tanks, can treat the entire flow of the plant.

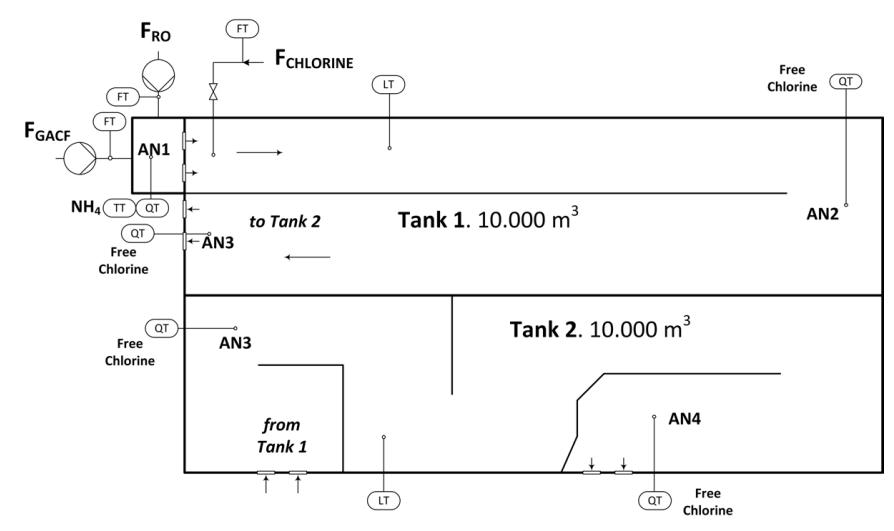

Fig. 1. Diagram of the chlorination plant that is used in this study

The flow of water to be treated in tank 1 comes from a small chamber in which water from a traditional treatment by granular activated carbon filters $(F G A C F)$ is mixed with water treated by reverse osmosis $(F R O)$. On output from both treatment processes, there are respective flow meters that show the flow from both the osmosis and carbon filter treatments, FRO and FGACF, respectively. The sum of these two flows will be the total flow to be treated variable, and the input for the control system presented in this article. The actual flow treated by the plant is between $5.0 \mathrm{~m}^{3} / \mathrm{s}$, at maximum performance, and a minimum flow in certain situations of $1.5 \mathrm{~m}^{3} / \mathrm{s}$. Under normal conditions, the distribution by $G A C F$ and by $R O$ is $50 \%$. However, this distribution may change by hours depending on the operating conditions of the DWTP. This flow balance must be taken into account by the control system because it will cause variations in the system's gain, as shall be explained later. The theoretical residence time in the tanks can vary between $30 \mathrm{~min}$ and 2.5 hours, depending on the flow. It is important for this time, depending on the velocity of the water, to be considered by the control system in order to modify its integral gain. The tank is divided by a baffle that forces the water to travel 200 meters, $182 \mathrm{~m}, 91$ in one direction and 91 $\mathrm{m}$. in the opposite direction. This design is due to the need to guarantee a minimum reaction time so that the dosage of chlorine that comes into contact with the water can be effective.

The sensors and actuators that are used to perform the control are flow meters (FT, flow transmitter) (FRO, FGACF), a quality transmitter $(\mathrm{Q})$, ammonia analyzer in the mixing chamber (AN1) and a temperature transmitter (TT) and level meters (LT, level transmitter) for tanks 1 and 2. Two free chlorine analyzers located at the midpoint (specifically at 87 $\mathrm{m})$ at the end of the tank (AN2, AN3, respectively). As actuators there are four chlorination cabins that produce a flow 
of chlorinated water (FCHLORINE) in accordance with the setpoint. Of the four cabins, two have a range of $0-40 \mathrm{Kg} / \mathrm{h}$ and the other two of $0-160 \mathrm{Kg} / \mathrm{h}$. The main meter that closes the loop from the control system in question is AN2, but there is a second meter (AN3) on output from tank 1.

\section{Control Strategy}

The main objective of the control system designed and implemented herein is to cover the basic demand for chlorine in tank 1 (Figure (1)). A control strategy with PI controller and feed-forward control from a fuzzy supervisor has been designed. This article describes the control for tank 1 .

Figure (2) shows a block diagram of the control implemented for tank 1. There is a fuzzy supervisor that controls a feed-forward block that generates two outputs (FF_MON, FF_NH4) in addition to the control signal generated by the PI controller. The fuzzy supervisor input variables are temperature, ammonium $\left(\mathrm{NH}_{4}+\right)$ in the mixing chamber, and the flow rates from reverse osmosis (FRO) and the granular activated carbon filters (FGACF). The setpoint (SCL) for the control system tends to be between $0.3 \mathrm{ppm}$ and $0.6 \mathrm{ppm}$ of free chlorine at output from tank 1 . This study has obtained four operating points (flows, $1.5 \mathrm{~m}^{3} / \mathrm{s}, 2.0 \mathrm{~m}^{3} / \mathrm{s}, 2.7 \mathrm{~m}^{3} / \mathrm{s}, 3.7 \mathrm{~m}^{3} / \mathrm{s}$ ) applying [31], depending on the possible range of flows to be treated, from $1.5 \mathrm{~m}^{3} / \mathrm{s}$ to $5.0 \mathrm{~m}^{3} / \mathrm{s}$.

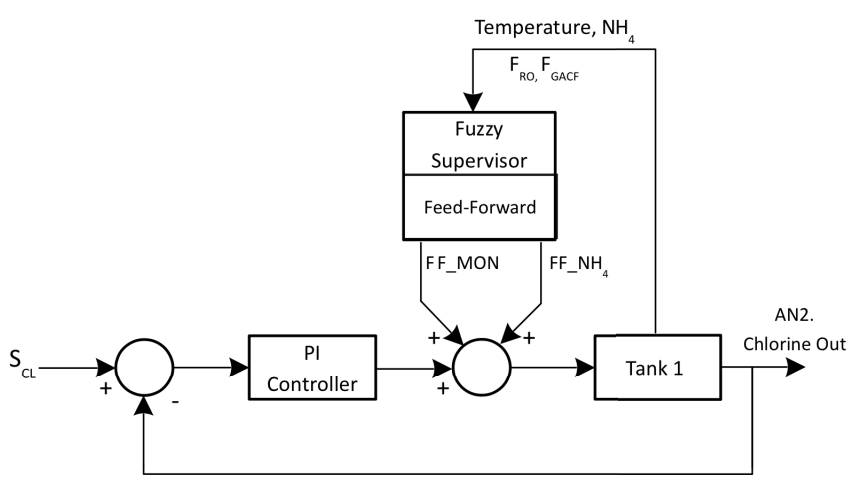

Fig. 2. Diagram of the proposed control scheme for the chlorination plant

\section{A. Feedback PI Control}

The basic feedback loop is based on a PI control system [11] [12]. This is already in place and will it is not the focus of this contribution. However, a succinct description is reported here just for providing a complete picture of the overall control system. The gains of the PI controller for the four described operating points were computed depending on the temperature in the mixing chamber and the water distribution between RO and FCAG. Based on historical data and with the help of the statistical tool $\mathrm{R}$ [32] it was possible to validate that the chlorine demand varies in winter and summer between $0.3 \mathrm{ppm}$ and $0.5 \mathrm{ppm}$ of chlorine due to temperature. This demand can drop to 0.15 for cases in which a large amount of the treated flow is from reverse osmosis, where a large
TABLE I

CONTROLLER GAINS FOR DIFFERENT OPERATING POINTS

\begin{tabular}{|c|c|c|c|}
\hline \multicolumn{2}{|c|}{ Operating Point } & \multicolumn{2}{c|}{ Controller } \\
\hline Chlorine Demand & Flow & $K_{p n}$ & $K_{i n}=K_{p n} T_{i n}$ \\
\hline $0.15 \mathrm{ppm}$ & $2.0 \mathrm{~m}^{3} / \mathrm{s}$ & $K_{p 1}=0.3$ & $K_{i 1}=0.00024$ \\
\hline $0.35 \mathrm{ppm}$ & $1.5 \mathrm{~m}^{3} / \mathrm{s}$ & $K_{p 2}=0.6$ & $K_{i 2}=0.00075$ \\
\hline $0.25 \mathrm{ppm}$ & $2.7 \mathrm{~m}^{3} / \mathrm{s}$ & $K_{p 3}=0.4$ & $K_{i 3}=0.0006$ \\
\hline $0.15 \mathrm{ppm}$ & $3.7 \mathrm{~m}^{3} / \mathrm{s}$ & $K_{p 4}=0.3$ & $K_{i 4}=0.00045$ \\
\hline
\end{tabular}

part of the organic and inorganic matter has been eliminated. The controller obeys to the typical Proportional-Integral (PI) formulation as:

$$
G_{c}(s)=K_{p n}\left(1+\frac{1}{T_{i n} s}\right)
$$

In equation (1), $K_{p n}$ is the proportional gain and $T_{i n}$ the integral time, where $n$ that has values $1,2,3$ and 4 for the different operating points. Table I presents a subset of the gains $\left(K_{p n}, K_{i n}\right)$ already in place.

\section{B. Fuzzy Feed-Forward Control}

The tool used in the design of the fuzzy supervisor was the Rockwell Automation RSLogix 5000 Fuzzy Designer commercial software [34]. The linguistic variables that were defined are Flow, Temperature, TPGacf and Amonium. Depending on the temperature and origin of the water, the control system makes an estimate of the demand for chlorine based on the organic matter in the water to be treated. This estimated chlorine demand (FF_MON) is a value that is added to the PI controller output variable. Depending on the amount of ammonium in the water to be treated, the control system computes a value, FF_NH4, which is added to the PI control output. Its value can vary between 0 and $7.375 \mathrm{ppm}$ depending on the ammonia in the water $(0 \ldots 1 \mathrm{ppm})$.

For example, figure (3) shows for the linguistic variable Flow with a universe of discourse $U=[1.0,5.0]$, the four fuzzy sets $(\mathrm{c} 1, \ldots, \mathrm{c} 4)$ with the respective linguistic values (labels) llow, low, medium and high with GAMMA and LAMBDA type membership functions. In the specific case, for a flow value of $3.2 \mathrm{~m}^{3} / \mathrm{s}$, the degrees of membership to the low and medium sets are 0.3 and 0.7 , respectively.

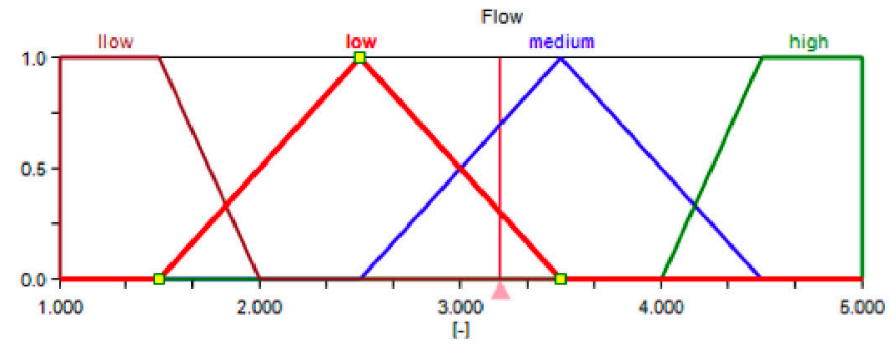

Fig. 3. Fuzzy sets (llow, low, medium, high) on the flow input variable, Flow [1..5] and a membership value of 0.18 at low and 0.82 at medium.

Table II shows a subset of the rules defined to interpolate the different gains of the control system. The defuzzification 
TABLE II

SUBSET OF DEFINED INFERENCE RULES FOR FF_MON

\begin{tabular}{|c|c|c|c|}
\hline \multicolumn{3}{|c|}{ If } & Then \\
\hline Flow & Temp. & TPGacf & FF_MON \\
\hline low & winter & normal & high \\
\hline medium & winter & normal & high \\
\hline medium & summer & normal & medium \\
\hline low & & low & low \\
\hline high & & high & high \\
\hline high & summer & normal & medium \\
\hline
\end{tabular}

algorithm applied was the center average method. The output variable FF_MON is classified into a singleton fuzzy set with vector $[0.15,0.25,0.35]$. Empirically, it has been verified, from 5-year historical data, that the chlorine demand in cases where there is no ammonia ranges between 0.15 and $0.35 \mathrm{ppm}$ of chlorine. The case of lower demand $(0.15 \mathrm{ppm})$ occurs when the flow from Reverse Osmosis (FRO) is predominant with respect to the flow from carbon filters (FGACF). In winter with a FGACF greater than $50 \%$ the demand can be around $0.35 \mathrm{ppm}$ and $0.25 \mathrm{ppm}$ in summer.

\section{Fuzzy Feed-Forward Ammonium Compensation Control}

The control system must compensate for severe disturbance in cases where ammonia is detected in the mixing chamber. There are currently ammonium analyzers $\left(N_{4} \quad N\right)$ on the market that have acceptable precision and accuracy with sample times of $5 \mathrm{~min}$. The problem with these analyzers is that below $0.02 \mathrm{ppm}$, their measurements are not reliable. This problem is solved by the designed control system, with the estimation of chlorine demand based on the temperature and origin of the water to be treated. As explained, there is a meter in the mixing chamber that enables the control system to detect the presence of ammonia and generate a proportional output in addition to the PI control action as shown in Figure (1). The meter output is used by the control system for values above $0.02 \mathrm{ppm}$ of $\mathrm{NH}_{4}$. For ammonium compensation, the control system uses a conventional Takagi-Sugeno model that offers a smooth change between the different slopes of action on the control variable.

Hourly values from the last 5 years have been analyzed and a linear correlation of 0.93 has been obtained between the ammonium meter readings and the chlorine demand. Equation (2) presents the obtained correlation. With the appearance of ammonia, the controller output due to FF_NH4 is updated by multiplying the existing ammonium value $\left(\mathrm{NH}_{4}\right)$ by the value slope of 7.37529 .

$$
y=7.37529 \mathrm{NH}_{4}+0.39694
$$

In equation (2) the value of the ordinate at origin is 0.39694 and is equivalent to the chlorine demand due to the organic and inorganic matter present. This value is already taken into account by the control system in its FF_MON term and is not added to the control variable in the finally
TABLE III

DEFINED INFERENCE RULES FOR THE OUTPUT VARIABLE FF_NH4

\begin{tabular}{|c|cc|}
\hline If & Then \\
\hline Ammonia IS Error & $y=0$ & \\
\hline Ammonia IS Good & $y=7.37529$ & $\mathrm{NH}_{4}$ \\
\hline
\end{tabular}

implemented control. Table III shows the two rules defined for the ammonia output variable.

\section{HARDWARE IMPLEMENTATION}

The existing control architecture in the DWTP is based on the ControlLogix system by Rockwell Automation [13]. User defined type (UDT) data structures were created to logically group the variables of the PI and Feed-Forward control system. Distributed throughout the DWTP they are linked to the PLCs that control the different processes (intermediate pumping, capture, chlorination, etc.). The SCADA supervisory layer that exchanges data with the controller is based on the System Platform solution by Wonderware [14]. Finally, the existing communication between the PLC controllers and the SCADA is based on the Ethernet/IP standard, Common Industrial Protocol (CIP).

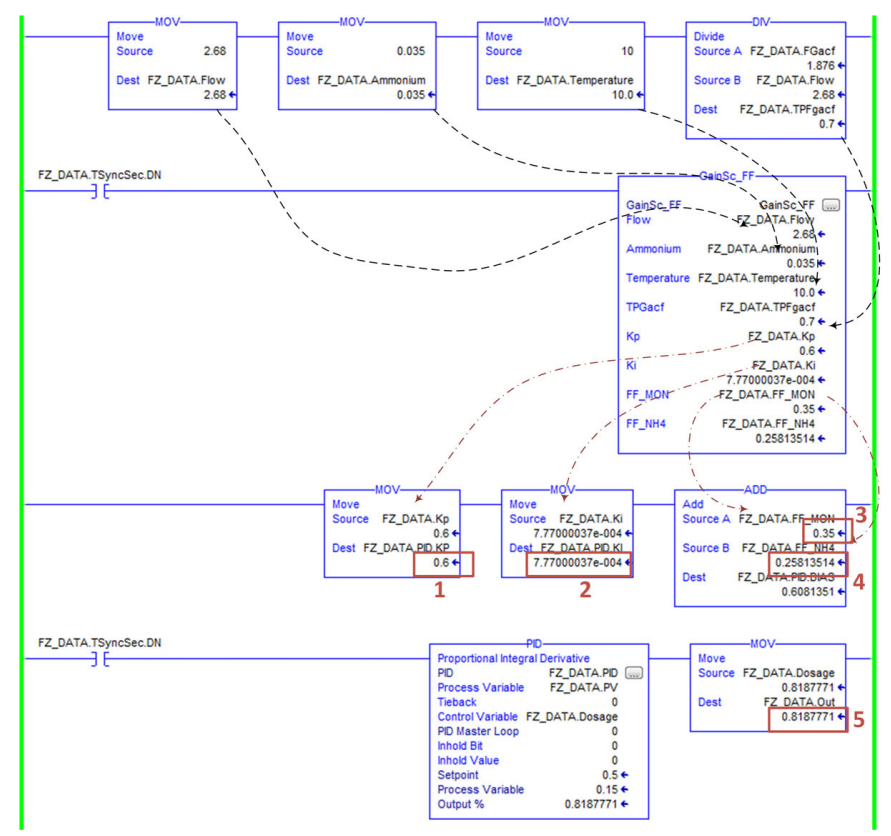

Fig. 4. Parameterization of the Fuzzy FF instruction and the PI Control

Figure (4) shows a fragment of the code run in the PLC controller. The setpoint, for this example, is $0.5 \mathrm{ppm}$ and the meter reading is $0.15 \mathrm{ppm}$ of free chlorine. As shown in the figure, in the first line of code we load the input variables of the fuzzy block (GainSc_FF), namely the flow, ammonia and temperature. The percentage of water from the FGACF carbon filters is also loaded based on the division of the FGACF flow between the total flow. All of the variables are 
loaded into a data structure (FZ_DATA) that was created to encapsulate all control system variables and parameters.

The control system was started dynamically and smoothly so as not to produce abrupt changes when switching from semi-automatic to automatic mode. The semi-automatic mode is the one in which the operator sets the amount of chlorine to be dispensed in ppm and the control system varies the $\mathrm{Kg} / \mathrm{h}$ to flowpacing. In the system start-up sequence, the operator sets the amount of chlorine to be dispensed (dSEMI) based on the state of the plant and his/her experience. After a preset stabilization time called T2End, the system switches from semi-automatic to automatic mode, the control variable then being controlled by the automatic control system system. During T2End the control system goes into a mode called Software-Manual and forces output of the PI controller to the dSEMI value. The T2End function is computed for the time when the control system system is in Software-Manual mode. This is obtained from the flow rate and the distance to the measuring point (i.e. where the AN2 sensor is located). Its purpose is to show when the system can switch to SoftwareAutomatic mode. Figure (5) shows part of the code of the function that is used for calculation of that T2End. For the case of the example, with a flow rate of $4.0 \mathrm{~m}^{3} / \mathrm{s}$, we get a velocity of $0.139 \mathrm{~m} / \mathrm{s}$ for the tank section at input. Every second the distance traveled by the sheet of water is increased and is adapted to possible variations in flow (velocity). Once the T2End time interval ends (T2End=0), i.e. the sheet of water has traveled the distance to the meter reading point, the system transfers control to the fuzzy supervisor, which goes from Software-Manual mode to what is called SoftwareAutomatic mode, where the PI updates its output based on the error and the value of the $\mathrm{Kp}$ and $\mathrm{Ki}$ gains of the controller.

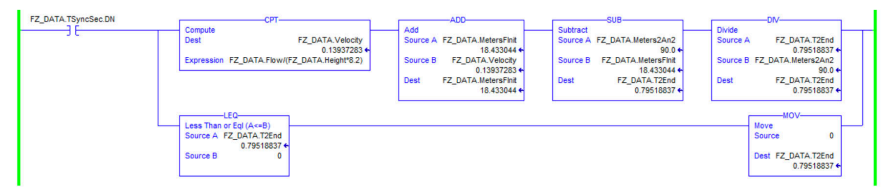

Fig. 5. Running the time calculation of the FF system in Software-Manual mode

\section{EXPERIMENTAL RESUlTS}

This section presents the results obtained both in the actual DWTP based on using the PI and Feed-Forward control system. Note that the graphs chosen are those that are considered most significant, bearing in mind the problems presented in the introduction section, variation in chlorine demand, flow variations and, consequently, delay times and the appearance of the ammonium disturbances, which can cause non-linear variations in demand. The following are graphs obtained with the Historian Client Trend tool of the SCADA in the DWTP in question. Three cases are presented that show the behaviour of the proposed controller in response to the problems raised in the introduction section. Table IV
TABLE IV

DESCRIPTION AND RANGE OF VARIABLES USED IN THE EXPERIMENTAL RESULTS

\begin{tabular}{|c|l|l|}
\hline Variable & Description & Range in Trend \\
\hline Flow & Total flow treated & $0 \ldots 5 \mathrm{~m}^{3} / \mathrm{s}$ \\
\hline AN2 & $\begin{array}{l}\text { Chlorine analyzer at } 87 \mathrm{me}- \\
\text { ters of the dosing }\end{array}$ & $0 \ldots 1 \mathrm{ppm}$ free chlorine \\
\hline AN3 & $\begin{array}{l}\text { Chlorine analyzer at } 178 \\
\text { meters of the dosing }\end{array}$ & $0 \ldots 1 \mathrm{ppm}$ free chlorine \\
\hline Set Point & Target value at output & $0 \ldots 1 \mathrm{ppm}$ free chlorine \\
\hline $\mathrm{NH}_{4}-N$ & $\begin{array}{l}\text { Ammonium analyzer at mix- } \\
\text { ing chamber }\end{array}$ & $0 \ldots 0.5 \mathrm{ppm} \mathrm{ammonium}$ \\
\hline FRO & Flow from reverse osmosis & $0 \ldots 5 \mathrm{~m}^{3} / \mathrm{s}$ \\
\hline FGACF & $\begin{array}{l}\text { Flow from granular carbon } \\
\text { active filters }\end{array}$ & $0 \ldots 5 \mathrm{~m}^{3} / \mathrm{s}$ \\
\hline HypoClean & $\begin{array}{l}\text { Flow of cleaning pump of } \\
\text { carbon filters }\end{array}$ & $0 \ldots 500 \mathrm{Hypochlorite}$ \\
\hline
\end{tabular}

shows a description and range of the variables used in the graphs.

Figure (6) shows an episode of a stepwise increase in ammonium where the proposed control system is able to act in advance and counteract the decrease at the outlet (AN2). Note that both AN2 and AN3 are always within the \pm 0.1 ppm interval of chlorine around the setpoint (which in this case is equal to $0.55 \mathrm{ppm}$ ).

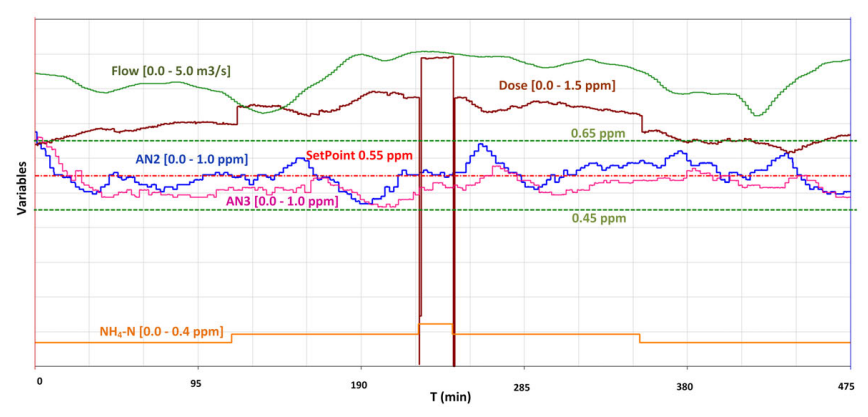

Fig. 6. PI and Feed-Forward controller's response to an ammonium episode from 0.03 to $0.05 \mathrm{ppm} \mathrm{NH}_{4}$

Figure (7) shows a typical situation of increase in flow that occurs when switching between treating water from aquifers and rivers. In a normal situation, the treated water is of fluvial origin; however, when the turbidity of the river is high due to the effect of rain, aquifer water is treated instead. When river water can be treated again, the DWTP begins to process this water. This effect can be observed in the graph that, for a short period of time, goes from treating $2 \mathrm{~m}^{3} / \mathrm{s}$ of aquifers to almost $5 \mathrm{~m}^{3} / \mathrm{s}$ of river water. The successive stepwise increase in FRO by $0.4 \mathrm{~m}^{3} / \mathrm{s}$ (the capacity of an RO frame) can also be observed, as can a gradual increase in water from carbon filters (FGACF). As can be seen, throughout this flow episode, a low but sufficient ammonium level remains constant enough for the control system to take it into account $\left(0.023 \mathrm{ppm}\right.$ of $\left.\mathrm{NH}_{4}-\mathrm{N}\right)$. Throughout the described action, the FF controller keeps the tank output (AN3) within the 
range of $0.1 \mathrm{ppm}$ around the setpoint.

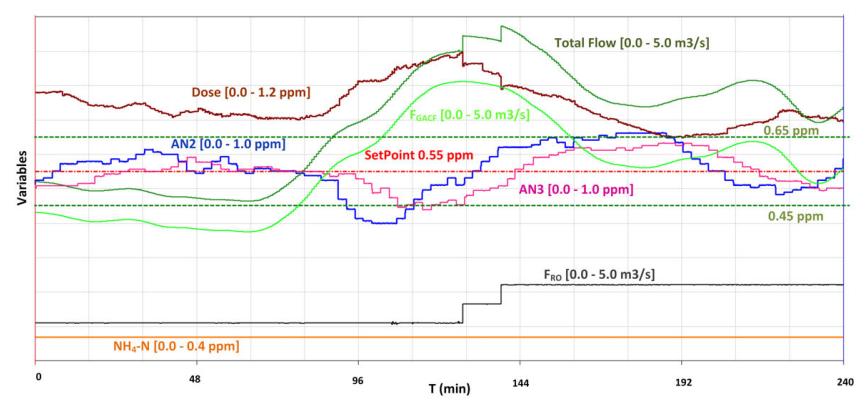

Fig. 7. Transient response of the control system to an increase in flow of more than $100 \%$.

Meanwhile, figure (8) represents an instance of using sodium hypochlorite to clean the carbon filters. This cleaning is done periodically depending how long the filter has been washing the filter. The effect caused to the system is a decrease in chlorine demand of approximately $0.2 \mathrm{ppm}$ of chlorine. Until minute 100 , the system maintains the chlorine output at the $0.55 \mathrm{ppm}$ setpoint. As shown, the FF system, after dosing hypochlorite and an increase in chlorine at the outlet, reacts and corrects its dosage to stabilize the chlorine as required. Throughout the episode of four continuous cleaning operations, the control system keeps the tank outlet (AN3) within the interval of $0.1 \mathrm{ppm}$ of chlorine around the setpoint (equal to $0.55 \mathrm{ppm}$ ).

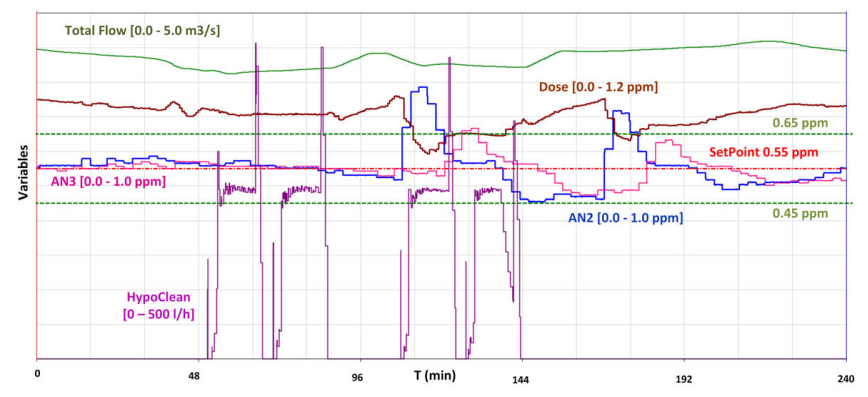

Fig. 8. PI and Feed-Forward controller's response to an ammonium episode from 0.03 to $0.05 \mathrm{ppm} \mathrm{NH}_{4}$

\section{CONCLUSIONS}

Chlorination systems in drinking water treatment plants (DWTP) can present different problems that make it difficult for control of chlorination in effluent to be effective. Long downtimes, variation in chlorine demand, or the occasional appearance of various nonlinear ammonia disturbances are just some of these problems that have a major effect on the design of the control system when it comes to automating the chlorination process. This process belongs to the group of distributed parameter systems where the phenomena of diffusion or convection arise and which must be taken into account both in the simulation and in the control.

This article has presented the analysis, design and implementation of a control system based on variable gains and feed-forward that addresses these problems. The article has described the design of the controller, explaining the control architecture, the set of fuzzy rules used, its implementation in a real system. Experimental results are obtained in a real treatment plant. The FF control has been compared with a conventional PI. The results show a considerable improvement to the system with respect to the use of a conventional PI. Stable output from the control system is guaranteed, within the expected margins in case of any variation in chlorine demand, flow rate or any possible disturbance such as the appearance of ammonia or cleaning with sodium hypochlorite.

\section{REFERENCES}

[1] A. Costán-Longares, M. Montemayor, A. Payán, J. Méndez, J. Jofre, R. Mujeriego, and F. Lucena, "Microbial indicators and pathogens: Removal, relationships and predictive capabilities in water reclamation facilities," Water Research, vol. 42, no. 17, pp. 4439-4448, nov 2008. [Online]. Available: https://linkinghub.elsevier.com/retrieve/pii/S0043135408003102

[2] R. Vilanova, R. Katebi, and N. Wahab, "N-Removal on Wastewater Treatment Plants: A Process Control Approach," Journal of Water Resource and Protection, vol. 3, no. 1, pp. 1-11, 2011.

[3] B. Dabkowski and M. White, "RTC-N : Modelling Ammonia-Based Aeration Control in Real Time for Deeper Process Control," $\mathrm{HACH}$ World Headquarters: Loveland, Colorado USA, 2015.

[4] Endress+Hauser, "Sludge treatment and disposaltle." [Online]. Available: https://www.endress.com/en/industry-expertise/waterwastewater/Sludge-treatment-and-disposal

[5] - "Automation of nitrogen removal from wastewater." [Online]. Available: https://www.endress.com/en/solutions-loweringcosts/analytical-solutions/automation-nitrogen-removal-wastewater

[6] Black \& Veatch Corporation, Whites Handbook of Chlorination and Alternative Disinfectants. Hoboken, NJ, USA: John Wiley \& Sons, Inc., dec 2009. [Online]. Available: http://doi.wiley.com/10.1002/9780470561331

[7] D. Gang, "Modeling Chlorine Decay in Surface Water," Journal of Environmental Informatics, vol. 1, no. 1, pp. 21-27, mar 2003. [Online]. Available: http://www.iseis.org/jei/abstract.asp?no=200300003

[8] R. M. Clark, "Chlorine Demand and TTHM Formation Kinetics: A Second-Order Model," Journal of Environmental Engineering, vol. 124, no. 1, pp. 16-24, jan 1998. [Online]. Available: http://ascelibrary.org/doi/10.1061/\%28ASCE\%290733$9372 \% 281998 \% 29124 \% 3 \mathrm{~A} 1 \% 2816 \% 29$

[9] E. A. Kobylinski, G. L. Hunter, and A. R. Shaw, "On Line Control Strategies for Disinfection Systems: Success and Failure," Proceedings of the Water Environment Federation, vol. 2006, no. 5, pp. 6371-6394, jan 2006. [Online]. Available: https://accesswater.org/publications/-293334/on-line-controlstrategies-for-disinfection-systems-success-and-failure

[10] E. A.-N. Furlong and F. M. D'Itri, "Trihalomethane levels in chlorinated Michigan drinking water," Ecological Modelling, vol. 32, no. 1-3, pp. 215-225, jun 1986. [Online]. Available: https://linkinghub.elsevier.com/retrieve/pii/0304380086900281

[11] R. Vilanova and A. Visioli, PID Control in the Third Millenium. Springer Verlag London Limited, 2012, pp. 207-234.

[12] H. S. Sanchez, F. Padula, A. Visioli, and R. Vilanova, "Tuning rules for robust fopid controllers based on multi-objective optimization with fopdt models," ISA Transactions, vol. 66, pp. 344 - 361, 2017.

[13] Allen-Bradley, "ControlLogix Control Systems." [Online]. Available: https://ab.rockwellautomation.com/ProgrammableControllers/ControlLogix

[14] AVEVA, "Wonderware solutions are now AVEVA solutions." 